\author{
Nota Científica \\ (Short Communication)
}

\title{
DISTRIBUCIÓN Y USO DE HÁBITAT DE THAMNOPHIS PULCHRILATUS (COPE, 1855) EN CHIGNAHUAPAN, PUEBLA, MÉXICO
}

\author{
DISTRIBUTION AND HABITAT USE OF THAMNOPHIS PULCHRILATUS (COPE, 1855) IN \\ CHIGNAHUAPAN, PUEBLA, MEXICO
}

Recibido: 26/11/2015; aceptado: 05/07/2016

González-Hernández, A., Moro-Hernández, D.M., \& Cruz, J. A. (2016). Distribution and habitat use of Thamnophis pulchrilatus (Cope, 1855) in Chignahuapan, Puebla, Mexico. Acta Zoológica Mexicana (n.s.), 32(3), 390-392.

ABSTRACT. We report the second record of Thamnophis pulchrilatus in the State of Puebla, and the first record of arboreal behavior for the species and second for the genus. In addition, we provide natural history data for this snake species.

Key words: snake.

Thamnophis pulchrilatus es una culebra listada endémica de México, con hábitos acuáticos para la búsqueda de alimento, conocida como culebra de agua en la región, de tamaño moderado con una LT de $772 \mathrm{~mm}$. Su distribución es irregular ocupando zonas montañosas de la Sierra Madre Occidental y Sierra Madre Oriental de 1,372 a 2,804 msnm, entre 18 y 24 grados de latitud norte (Dixon \& Lemos-Espinal, 2010). Lazcano-Villarreal et al., (2010) mencionaron que se distribuye en poblaciones disyuntas en Durango, Zacatecas, Nayarit, Nuevo León, Tamaulipas, Aguascalientes, Jalisco, Guanajuato, Querétaro, Hidalgo, Estado de México, Distrito Federal, Tlaxcala, Puebla, Veracruz y Oaxaca, mientras que Ramírez-Bautista et al., (2009) incluyeron además a los estados de Michoacán, Morelos y San Luis Potosí. Para el estado de Puebla sólo se reporta en la Faja Volcánica Transmexicana (García-Vázquez et al., 2009). Habita bosques de Oyamel, pino, pino-encino y roble (Lizárraga, 2006; Dixon \& Lemos-Espinal, 2010). Se conoce muy poco sobre la historia natural de T. pulchrilatus, Carbajal-Márquez et al., (2014) documentaron el comportamiento defensivo de la especie, pero aún no se conocen datos sobre su reproducción, cortejo o depredadores. En cuanto a su dieta Dixon \& Lemos-Espinal (2010) mencionaron que se desconoce, pero creen que es similar a otras culebras de agua que vi- ven en montañas, las cuales se alimentan principalmente de peces, larvas y adultos de anuros, sanguijuelas, lombrices de tierra, cangrejos y acociles. Ramírez-Bautista et al., (2014) mencionaron que también se alimenta de renacuajos y de algunos ejemplares adultos de Dryophytes eximius e Dryophytes plicatus. Habita en áreas elevadas, se cree que es mayormente diurna o crepuscular y está más adaptada al ambiente terrestre, es vivípara y está asociada a cuerpos de agua y sus secreciones orales tiene toxinas que paralizan a sus presas (Lazcano-Villareal et al., 2010).

Durante un muestreo de herpetofauna en el municipio de Chignahuapan, Puebla (1949’30.35'” N - 9759'25', O; WGS84). Se registraron dos ejemplares de Thamnophis pulchrilatus sobre árboles de encino (Quercus crassifolia) a una altura de $2.10 \mathrm{~m}$ y $4.96 \mathrm{~m}$ (Fig. 1). Los organismos se encontraban posando en las bifurcaciones de los árboles, un ejemplar en la bifurcación primaria y el otro en una bifurcación secundaria, ambos organismos se encontraban perchados sin actividad alguna y comenzaron a huir al acercarse a observarlas. El comportamiento arborícola podría estar asociado a la alimentación de anfibios y reptiles como lo sugiere Lizárraga (2006), en la zona se han observado como especies arborícolas a las lagartijas Abronia graminea (escorpión arborícola), Sceloporus microlepidotus (lagartija de mezquite) y Lepidophyma sylvaticum (lagartija nocturna de montaña), en la zona también se observaron los anuros Dryophytes eximius (ranita de montaña) y Rheohyla (Calate arborícola) que podrían formar parte del alimento de T. pulchrilatus.

El registro de esta especie es el primero para el municipio de Chignahuapan, Puebla, a 37 km en línea aérea de Mimiahuapan, Tlaxco, en el estado de Tlaxcala (19³7' $\mathrm{N}$ y $98^{\circ} 02^{\prime} \mathrm{W}, 3,054 \mathrm{msnm}$ ), el sitio más cercano con la presencia de Thamnophis pulchrilatus (Fig. 2) y es el 


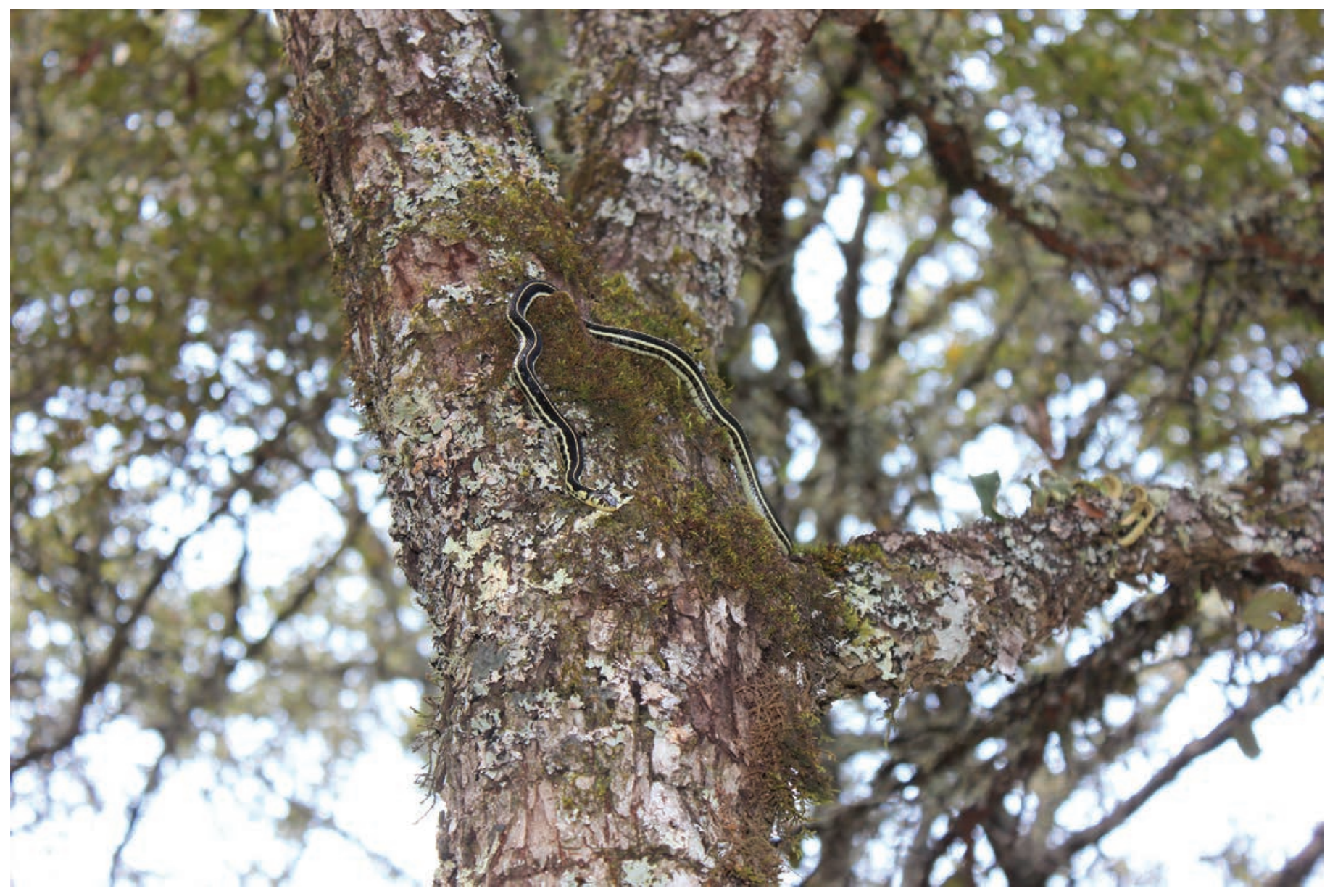

Figura 1. Ejemplar de Thamnophis pulchrilatus observado en árbol de encino a 4.96 m del suelo. Foto: Misael Casas.
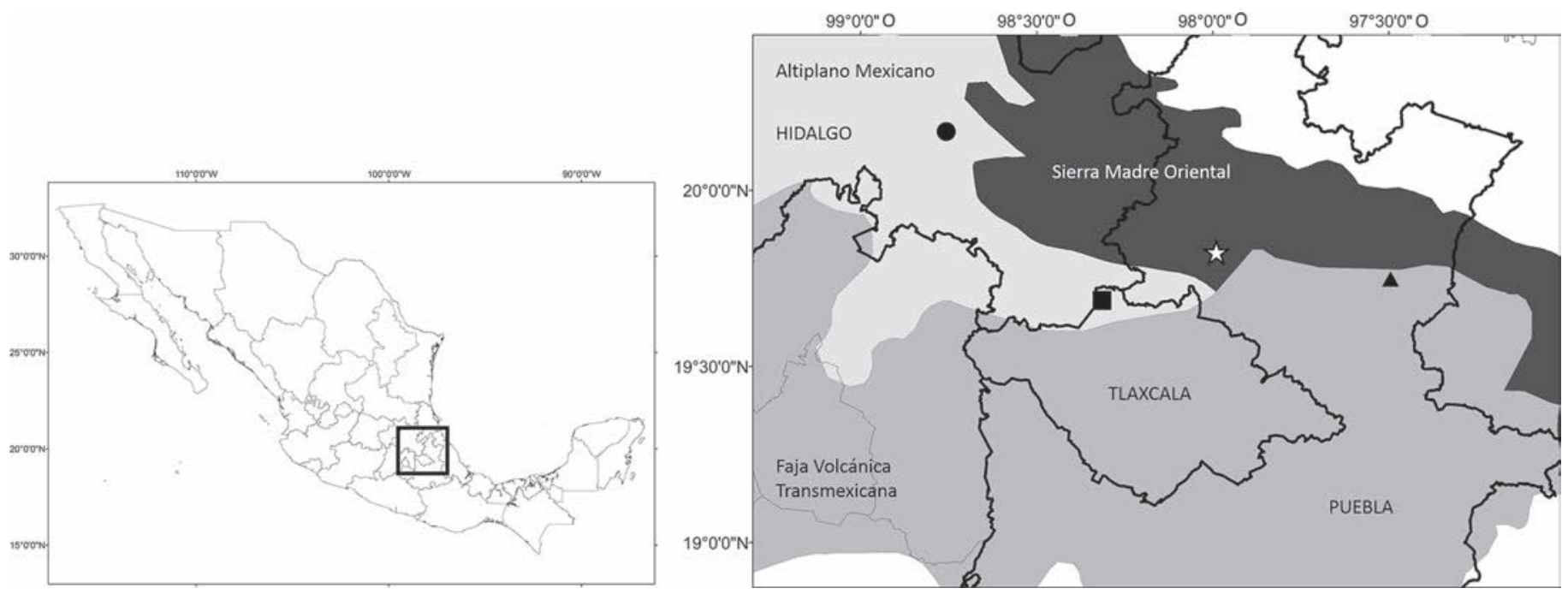

Figura 2. Mapa de distribución de Thamnophis pulchrilatus. Localidades: Chignahuapan, Puebla (estrella blanca); La Estanzuela, Mineral El Chico (ć́rculo negro); Mimiahuapan, Tlaxco (cuadro negro); Tlatlahuquitepec (triángulo negro). 
segundo reporte de la especie para el estado de Puebla. Además, es el primer reporte de hábitos arborícolas para T. pulchrilatus en México, contrario a lo mencionado por Lazcano et al., (2010) quienes indicaron que es una especie más adaptada al ambiente terrestre que otros miembros del género. Se registra la máxima altura $(4.96 \mathrm{~m})$ sobre un árbol, a la que se ha encontrado un ejemplar del género Thamnophis reportada en la literatura, difiriendo de los $1.5 \mathrm{~m}$ y $1 \mathrm{~m}$ de altura sobre el nivel del suelo que se ha reportado para T. sirtalis (Galbraith, 2001; Gilhen \& Strum, 2007). Actualmente, sólo se conoce este comportamiento arborícola dentro del género para $T$. sirtalis, en Canadá (Gregory, 1975; Galbraith, 2001; Shine et al., 2005; Gilhen \& Strum, 2007). El que una serpiente de agua del género Thamnophis presente un comportamiento arborícola se ha atribuido a comportamientos de cortejo (Galbraith, 2011; Gihen \& Strum, 2007), como parte de la actividad de la crías durante la primavera (Gregory, 1975) o como un comportamiento termorregulador (Shine et al., 2005).

\section{LITERATURA CITADA}

Carbajal-Márquez, R. A., González-Saucedo, Z. Y., \& G. E. Quintero-Díaz. (2014). Thamnophis pulchrilatus. Diet and defensive behavior. Herpetological Review, 45(2), 344

Dixon, J. R., \& J. A. Lemos-Espinal. (2010). Anfibios y reptiles del estado de Querétaro. Universidad de Texas, UNAM, CONABIO., $428 \mathrm{pp}$.

García-Vázquez O. U., Canseco-Márquez, L., Gutiérrez-Mayén, G., \& M. Trujano-Ortega. (2009). Actualización del conocimiento de la fauna herpetológica en el estado de Puebla, México. Boletín de la Sociedad Herpetológica Mexicana, 17(1), 12-36.

Galbraith, D. A. (2001). Arboreal courtship behavior by eastern gar- ter snakes, Thamnophis sirtalis sirtalis, in September in Bruce County, Ontario, Canadian Field-Naturalist, 115, 347-348.

Gilhen, J., \& Strum, R. (2007). Arboreal late summer courtship behaviour of maritime garter snake, Thamnophis sirtalis pallidulus, in Dartmouthm Nova Scotia, Canada. Canadian Field-Naturalist, 121, 210-211.

Gregory, P. T. (1975). Arboreal mating behavior in the red-sided garter snake. Canadian Field-Naturalist, 89, 461-462.

Lazcano-Villarreal D., Banda-Leal J., \& Jacobo-Galván, R. D. (2010). Serpientes de Nuevo León. Universidad Autónoma de Nuevo León, Monterrey, México. 502 pp.

Lizárraga, V. A. (2006). Tendencias evolutivas de algunos aspectos reproductores y tipo de alimentación en las serpientes del Género Thamnophis. Tesis Posgrado en Ciencias Biológicas. Instituto de Biología, UNAM.

Ramírez-Bautista A., Hernández-Salinas U., García-Vázquez U. O., Leyte-Manríquez A., \& Canseco-Márquez L. (2009). Herpetofauna del Valle de México: Distribución y Conservación. UAEH, CONABIO, México. 213 pp.

Ramírez-Bautista A., Hernández-Salinas U., Cruz-Elizalde R., Berriozabal-Islas Ch., Lara-Tufiño D., Goyenechea MayerGoyenechea, I., \& Castillo-Cerón J. M. (2014). Los anfibios y reptiles de Hidalgo, México: Diversidad, biogeografía y conservación. Sociedad Herpetológica Mexicana, México. 386 pp.

Shine, R., Wall. M., Langkilde, T., \& Mason, R. T. (2005). Scaling the heights: thermally driven arboreality in garter snakes. Journal of Thermal Biology, 30, 179-185.

\section{Adriana GONZÁLEZ-HERNÁNDEZ*, Dulce M. MORO-HERNÁNDEZ \& J. Alberto CRUZ}

Colección Nacional de Anfibios y Reptiles, Instituto de Biología, Departamento de Zoología, Universidad Nacional Autónoma de México, Circuito Exterior s/n, Ciudad Universitaria, Coyoacán C.P. 04510, México, D.F.

* Autor de correspondencia: <adricnar@yahoo.com.mx> Editor responsable: Gustavo Aguirre León. 